\title{
BMJ Open GPs' views and experiences of managing patients with personality disorder: a qualitative interview study
}

\author{
Lydia French, Paul Moran, Nicola Wiles, David Kessler, Katrina M Turner
}

To cite: French L, Moran $\mathrm{P}$, Wiles N, et al. GPs' views and experiences of managing patients with personality disorder: a qualitative interview study. BMJ Open 2019;9:e026616. doi:10.1136/ bmjopen-2018-026616

- Prepublication history for this paper is available online. To view these files, please visit the journal online (http://dx.doi. org/10.1136/bmjopen-2018026616).

Received 11 September 2018 Revised 12 December 2018 Accepted 8 January 2019

\section{Check for updates}

(c) Author(s) (or their employer(s)) 2019. Re-use permitted under CC BY-NC. No commercial re-use. See rights and permissions. Published by BMJ.

Population Health Sciences, Bristol Medical School, University of Bristol, Bristol, UK

Correspondence to

Dr Lydia French;

lydia.french@bristol.ac.uk

\section{ABSTRACT}

Objective The aim of this study was to explore GPs' views and experiences of managing patients with personality disorder (PD), and their views on the role of the Improving Access to Psychological Therapies (IAPT) programme as a support service.

Design In-depth interviews, analysed thematically. Participants Fifteen GPs (7 women and 8 men) of varying age and clinical experience, working in practices that differed in terms of the socio-demographic characteristics of their patient populations.

Setting Twelve general practices based in the West of England, UK.

Results GPs described patients with PD as being challenging to work with and that this work required dedicated time and care. They described experiencing particular difficulty with monitoring their patient's mental health, as well as having little knowledge about the efficacy or availability of treatments for their attenders with PD. They were aware that this patient population often experienced poor mental health and reported a propensity for them to fall into the gap between primary and secondary mental health services, leaving GPs with little choice but to improvise their own management plans, which occasionally involved funding third-sector treatment options. In terms of IAPT services' role in managing these patients, GPs wanted shorter waiting times, better interagency communication, more highly trained therapists and more treatment options for patients with PD. They also wanted the service to be able to 'hold' patients with PD in treatment over a longer period than currently offered. Conclusions Findings suggest that, overall, GPs did not consider National Health Service mental health services to offer an effective treatment for patients with PD. While they considered the IAPT programme to be a valuable service for patients with less complex mental health needs, such as depression and anxiety; they felt that the current service provision struggled to meet the needs of patients with more complex mental health needs, as exemplified by people with $\mathrm{PD}$.

\section{BACKGROUND}

People with personality disorder (PD) have pathological personality traits accompanied by impairments in interpersonal functioning. ${ }^{1}$ About $4 \%$ of the general population meet diagnostic criteria for $\mathrm{PD} ;{ }^{2}$ although prevalence is much higher among primary

\section{Strengths and limitations of this study}

This is the first study to detail GPs' views and experiences of managing patients with personality disorder (PD), and their views on Improving Access to Psychological Therapies (IAPT) services in relation to meeting the mental health needs of these patients.

- Data were collected using in-depth interviews that enabled GPs to raise issues that were salient to them.

- The GPs interviewed were able to refer patients with PDs and depression to local IAPT services, but not all IAPT services in the UK accept these patients. Thus, our participants may have held views about the role of IAPT services that differ from those held by GPS working in other areas.

- The diagnosis of PD is usually made in secondary care and, it is possible that GPs were speaking about patients who did not have a formal diagnosis of PD.

care attenders ${ }^{3}$ and mental health service users. ${ }^{4} \mathrm{PD}$ is associated with poor physical and mental health, and substantial healthcare costs at the level of primary and secondary care. $^{5-11}$

The treatment of people with PD remains one of the most challenging areas in mental health, primarily because individuals with the disorder manifest their main problems when interacting with others, including healthcare practitioners. Indeed, research shows both clinicians and mental health workers find managing patients with PD difficult. ${ }^{12} 13$ PD affects the practitioner-patient relationship, as misunderstandings and difficult exchanges between patients and healthcare practitioners are not uncommon, and consistency, clarity and forward planning are all important in managing the relationship. ${ }^{14}$ In fact, the term PD has often been used in a pejorative sense, as a diagnosis of exclusion. Consequently, attention to the condition has vacillated between attempts to dismiss it as a non-diagnosis, or to regard it as a specialist subject in psychiatry deemed outside the 
scope of mental illnesses, GPs might be expected to identify and treat. ${ }^{15}$

Primary care is the frontline of service delivery in the National Health Service, but how GPs should manage these patients remains unclear due to the lack of evidence about which treatment or management approach would be most effective. ${ }^{16}$ We also know very little about how GPs currently manage these patients as, to date, no study has explored their views and experiences of doing so. Clinicians and mental health workers find managing patients with PD difficult, ${ }^{12} 13$ and that GPs might view the management of $\mathrm{PD}$ as beyond the scope of primary care and requiring specialist services. ${ }^{15}$

The main referral option for GPs for patients with PD and depression is a referral to psychological services-via the Improving Access to Psychological Therapies (IAPT) programme. This programme was established in England in 2008 to improve patient access to psychological interventions, in particular to cognitive behavioural therapy (CBT). The programme was expanded in 2011 to include the treatment of individuals with depression and anxiety, and co-morbid PD, ${ }^{17}$ but this expansion was not accompanied by additional training for IAPT practitioners in how to manage this complex patient group. It was also not accompanied by evidence supporting the effectiveness or feasibility of treating people with PD in this setting. Two recent national reports indicate that only $46.6 \%$ of those entering IAPT services achieve recovery status at the end of treatment, ${ }^{18}$ and there is evidence that variance in recovery status is partly explained by the presence of personality pathology. ${ }^{19}$

To determine how these patients should be managed, and whether primary care is a context within which this can be done, the aim of this study was to explore GPs' views and experiences of managing patients with $\mathrm{PD}$, and their views on the role of the IAPT programme as a support service.

\section{METHODS}

\section{Patient and public involvement}

As part of a wider study aiming to identify the best treatment for patients with PDs attending IAPT services, the study was discussed with three individuals diagnosed with PD. They provided feedback on the aims of the study, its design and study documents, including patient information sheets and topic guides.

\section{GP telephone interviews}

GPs were informed about the study by two Central Research Networks (CRNs) based in the West of England between May 2017 and August 2017. The CRNs were asked to approach practices registered with them that varied in terms of the socio-demographic characteristics of their patient populations.

Sixty-four practices were approached and 19 agreed to take part. Within the 19 participating practices, 22 GPs agreed to be interviewed. From these, we purposefully sampled 15 GPs of varying age and gender, working within 12 practices that differed in terms of the socio-demographic characteristics of their patient populations. Data collection continued until saturation of key themes was reached, that is no new themes were identified in the later interviews. The interviews were held by telephone, to encourage practitioner participation, and because well-planned telephone interviews can gather the same material as those held face-to-face. ${ }^{20}$

The interviews were conducted by an experienced qualitative researcher (Lydia French) who was not known to the study participants. A topic guide was used to ensure consistency across the interviews. The guide was based on the aims of the research, a review of relevant literature and the research team's knowledge of PD and the IAPT programme. The guide included questions about GPs' views and experiences of managing patients with PD, how they felt mental health services responded to the patient group, whether they thought it was appropriate for this patient group to be managed within IAPT services and what they thought an effective mental health service would need to offer these patients. The interviews were held between 01 May 2017 and 01 October 2017, and lasted between 20 and $30 \mathrm{~min}$.

\section{Data analysis}

Data collection and analysis proceeded in parallel, so that insights from the initial interviews could inform later data collection. Interviews were audio-recorded, fully transcribed and analysed thematically in accordance with the process outlined by Braun and Clarke ${ }^{21}$. Initially, Lydia French and Katrina Turner independently read a sample of transcripts to identify emerging themes and to develop a preliminary coding frame. They then met to discuss their coding. Once the coding frame had been agreed, transcripts were uploaded to $\mathrm{NVivo}^{22}$ and electronically coded. The researchers then read and re-read data under each code to identify key themes and deviant cases.

\section{RESULTS}

The characteristics of the 15 GPs interviewed are detailed in table 1. Below, themes relating to GPs' views and experiences of managing patients with PD are reported before their views on IAPT.

\section{GPs' experiences of managing patients with PD}

Most GPs interviewed described patients with PD in negative terms, using adjectives, such as 'dislikeable' and 'manipulative'. They portrayed them as living chaotic lives with a history of substantial adversity across their lives, including child abuse, domestic violence and major financial difficulties.

I guess personality difficulties are those people who have a background that suggests sort of a chaotic or difficult lifestyle that have had presumably, difficult childhood, lots of drug changes, drug and alcohol misuse, they have chaotic lifestyles with lots of 
Table 1 Socio-demographic description of the GPs interviewed in the study and their associated general practices

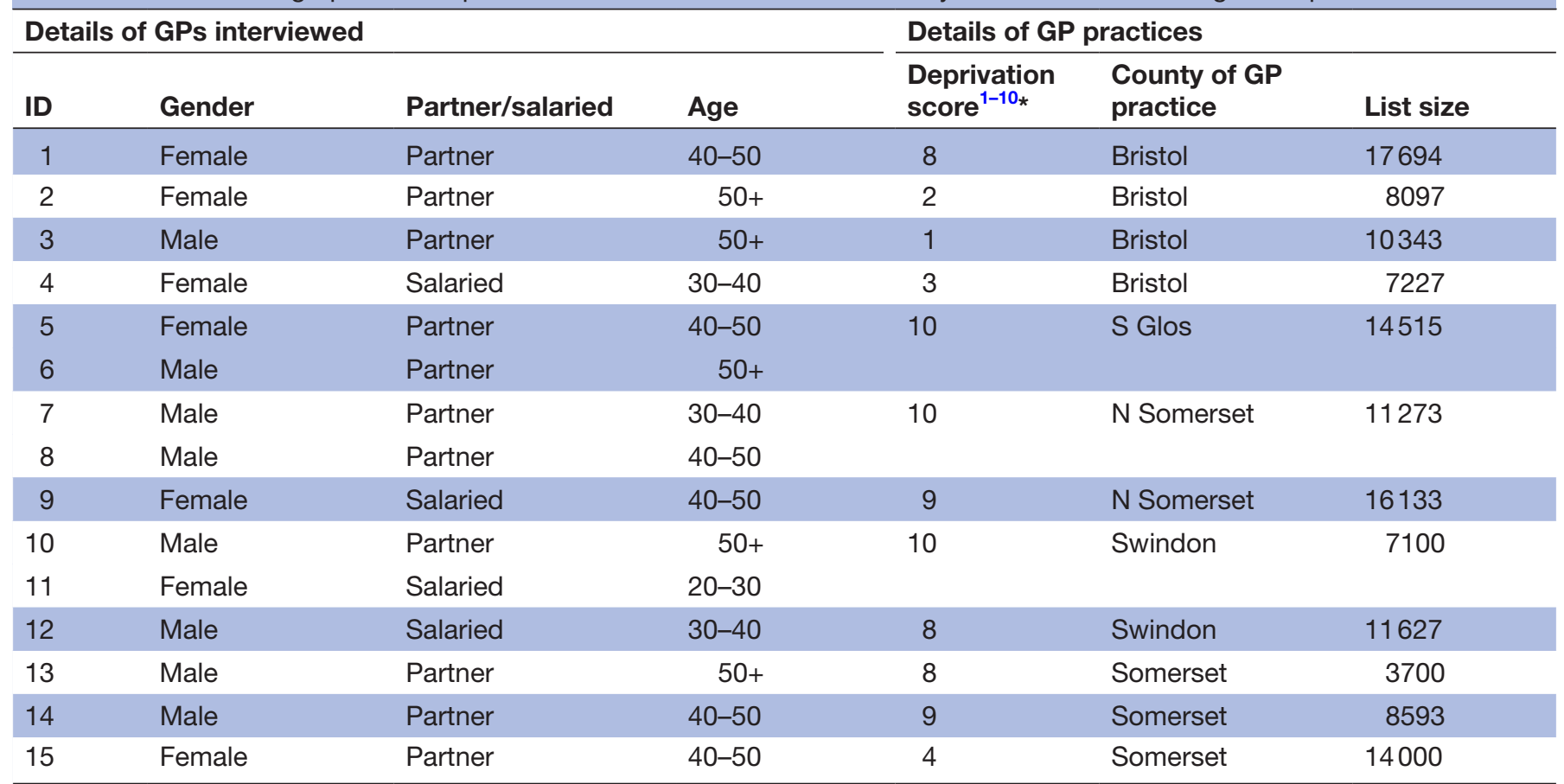

*Deprivation score for the practice patient population where one indicates the most deprived patient population and 10 the least deprived.

terminations of pregnancies so often there's a background to their behaviour really and I guess that kind of alerts you to the kind of person that you're dealing with. GP 3, Male

GPs described consultations with patients who had PD as unpredictable, challenging and requiring careful management in terms of how they communicated with the patient and how regularly they saw the patient. They depicted them as having multiple, complex health complaints, which they often struggled to communicate with the GP.

I think they're challenging, I try not to think of them as a problem group but you never really know where you are with them or what you are actually dealing with because they can be very bad at communicating the problem and because there are often lots of physical and mental health complaints it is hard to know where to begin and what to treat first. GP 11, Female

\section{Challenging consultation behaviour}

GPs reported patients with PD to be frequent attenders who were often unaware of the amount of practice time they required compared with other patients. They also described them as attending cyclically and typically at times of personal crisis, when other services had discharged them from care. Yet many GPs also described their patients with PD as regularly missing appointments and not engaging with health and social care services when referred for further investigation, treatment or support.
So, a classic example would be the lady I spoke about earlier. She doesn't really engage in the mental health services that she's offered so she has-she repeatedly presents to us, to A\&E, calling the CRISIS team and then doesn't engage in any of the package of help that she's offered. She just seems to bounce from one crisis to the next, but I suppose that is the nature of the condition. GP 1, Female

As a consequence of this erratic attendance, GPs found it difficult to track patients' health over the longer term, and to assess their level of risk.

It's difficult. We end up having to assume that if there is a problem they will attend and if there isn't they won't. If a patient doesn't turn up, which happens a lot, we really have no idea how they are or how at risk they might be. GP 7, Male

\section{The challenge of treating in primary care}

GPs described multiple challenges when trying to meet the needs of patients with PD, including having little knowledge about PD; having little knowledge about the efficacy or availability of talking therapies in primary and secondary care for patients with PD, and of not knowing which mental healthcare services to refer patients with PD for further treatment due to changing guidelines.

Referrals being knocked back and patients falling in the gaps between services

All the GPs interviewed described the propensity for patients with PD to fall in the gap between primary and 
secondary mental health services. GPs described multiple instances of referring patients to secondary care mental health services only for the referral to be rejected by the service on grounds that the patient was not deemed to be 'risky' enough to warrant treatment by a community mental health team. GPs also described patients with PD and poor mental health being refused treatment by primary care mental health services, including IAPT, because the patient was deemed to be at too high a risk of suicide or serious self-harm to be taken on for treatment. Faced with this dilemma, several GPs described adopting a strategy of 'writing-up', or embellishing descriptions of a patient's risk status in order to ensure that the patient was seen by secondary care services. On the other hand, faced with an overly cautious response from IAPT, several GPs described emphasising the patient's more 'agreeable' mental health conditions, such as depression or anxiety, to maximise the chances of the patient being accepted into treatment.

You know if you mention PD there will be nowhere at all for them to go so I'm usually very careful not to put it down in their notes. I usually say depressed or a bit anxious. Something that won't make them think the patient is risky. It's about knowing the hoops that you've got to jump through. GP 4, Female

Other GPs described employing additional mental health nurses or therapists to work within the practice to treat patients with PD and depression, funding private specialist mental health services to support the practice, or of trying to find charitable mental health services that might be willing to accept patients with PD.

...so, if they fall into a particular box they can access counselling and ongoing support but the trick is trying to find something that each person can fit into their box so that might be through a local church, it might be through the carers groups that we run here or the carer's society so unfortunately some people fall through, they don't meet any of the particular groups. GP 6, Male

However, despite these efforts, it was evident that the majority of GPs had developed their own ways of managing patients with PD. Many GPs described the importance of accepting that patients with PD need intensive, longterm management and psychological support and that it is highly unlikely that any treatment alone will provide a solution or 'quick fix'. All the GPs felt that it was important to develop a good relationship with the patient and this was best done by setting clear boundaries with the patient about attendance and contacting the surgery, that is set a weekly or monthly date for the patient to see the same clinician. GPs also spoke of the value of consulting other mental health experts and meeting weekly with the practice team in order to discuss and agree on treatment plans or management techniques for the more complex PD patients.

\section{GPs' views on IAPT for patients with PD}

Overall, GPs described IAPT as a useful service for patients with 'simpler' mental health conditions, such as mild to moderate depression or anxiety, who were willing and able to engage with treatment. However, when describing the helpfulness of IAPT for patients with PD and depression, GPs provided more negative accounts.

\section{PD patients need a service that can 'hold' and manage long- term risk}

GPs felt IAPT services offered too few sessions, leaving patients feeling abandoned at the end of treatment with unresolved mental health problems. They felt it was important that any mental health service for patients with PD should be able to contain and 'hold' the patient in treatment for longer, to monitor the patients' risk or selfharm or suicide.

Ultimately, we just need a service that can hold these kinds of patients. Be there on the end of a telephone when they have a crisis, be a point of contact. My gut feeling would be that these patients are just more complicated, and they need a more in-depth therapeutic relationship with a psychologist on an on-going basis not six sessions of CBT. GP 15, Female

\section{Patients with PD wait too long to be seen}

GPs also described patients with PD as having to endure particularly long waits, before being seen-waiting times that often far exceeded those experienced by patients with other mental health problems. Indeed, several GPs described patients with PD having to wait over 12 months for treatment. They felt shortening waiting times would reduce the likelihood of mental health problems escalating or patients disengaging from the health service altogether.

...the wait is part of the problem. If it was something a bit sooner, the ball had just started to roll and they get their psychological therapy fairly quickly you would maybe find it easier to get them to engage and problems wouldn't escalate as they have a tendency to do. GP 7, Male

\section{IAPT does not engage patients with PD due to pressures on the service}

Interviewees felt that due to the very large numbers of patients referred to IAPT services, these services did not actively try to engage patients with PD in therapy. This was particularly problematic for this group, as GPs felt these patients were sensitive to rejection and needed encouragement to stay engaged with services. Furthermore, GPs felt that in order to manage referral numbers, IAPT was increasingly offering patients 'group therapy' rather than 'one-to-one therapy'. Most of the interviewees felt this would not appeal to patients with PD, who, they felt, may not want to share their feelings in a group setting. Indeed, many of the 
GPs described the difficulty they had experienced in getting patients to take up the offer of group therapy. Nevertheless, one GP considered 'group therapy' to be helpful, as the group format could lend valuable social support to the patient and counteract feelings of isolation in the context of their distress.

\section{GPs want better communication with IAPT}

More than half of the GPs interviewed reported only hearing from IAPT when their patient had not engaged in the service. Many of the GPs felt that good communication between a mental health service for patients and the GP was essential within a 'shared care' framework, and this was particularly pertinent for PD patients who often had multiple health problems requiring input from multiple agencies.

We get very little feedback from the talk therapies team as to how they felt things went. We obviously get the feedback oh they attended six out of the seven sessions or you know, initially they seem very depressed, but you never get a feedback as to how treatment is going. It's important I feel because if the service works closely with you it helps you to support the patient better. Added to which many of these patients have other health complaints and better communication can only lead to better treatment. GP 1, Female

\section{The need for skilled therapists}

GPs felt that there were not enough trained staff within IAPT who could manage more complex patients. This was reported as resulting in patients being turned away.

I get people rejected all the time. So, I had a girl yesterday who was rejected because her mental health needs were too complicated. She is complicated, and she's got risk going on and they won't see her because the therapists aren't really trained to cope with risky patients, don't really know how to manage her. GP 10, Male

\section{GPs want better treatments for patients with PD}

While most GPs expressed having little knowledge about how best to treat patients with PD, many felt that CBT was simply not designed for this patient group and highly unlikely to be effective due to their short term, inflexible nature' (GP 2, Female). Indeed, across the interviews, it was clear that GPs thought the most effective treatment for this patient group would be one that allowed patients time to speak about past experiences.

...to my mind these patients need listening to. They have often been through so much in their lives and they need to lay it all out on the table and be heard for the first time. I feel it is an important part of their recovery that they are able to talk about the things that have happened to them. GP 10, Male
GPs also thought that the treatment given needed to help patients with PD gain mastery over their emotions and relationships with others. GPs described it as necessary for any potential therapy to 'educate' and 'up-skill' the patient in managing their emotional and behavioural responses to other people and life events.

\section{DISCUSSION}

The findings from our study suggest that GPs find patients with PD challenging, akin to Groves' notion of the 'hateful patient' that he considers fill clinicians with dread (1978). ${ }^{23}$ This finding is supported by previous research that shows both clinicians and mental health workers find managing patients with PD difficult. ${ }^{12}{ }^{14}$ GPs in our study also reported patients with PD to be time-consuming to manage. There is a paucity of research concerning the management of PD in primary care. However, findings by Dubovsky and Kiefer, ${ }^{24}$ who explored the management of borderline PD in primary care, suggest that the doctorpatient relationship can be greatly improved if the physician has a good understanding of the disorder and good communication with all the providers involved in the patient's care.

Moreover, the evidence base for the effective treatment of PD is insufficient. ${ }^{1516}$ It is, therefore, unsurprising that GPs reported having little knowledge about PD or being able to effectively treat it. GPs also considered patients with PD to have complex health and social needs, manifesting in challenging consultations that required careful management.

GPs also felt that the needs of these patients were not being fully met either by themselves or by IAPT services. GPs were particularly frustrated by the propensity for patients with PD and poor mental health to fall in the gap between primary and secondary mental health services, leaving them with full responsibility for care and treatment of these patients. Indeed, GPs' accounts point to the absence of a mental health service that could 'hold' and provide long-term risk management for patients with PD. This perhaps reflects the current changing organisation of healthcare, which according to Bateman et al. ${ }^{25}$ has resulted in the closure of inpatient services in secondary care and the delivery of ever shorter, more infrequent treatments being delivered in primary care.

While the government has expanded the scope of IAPT services to include the treatment of individuals with depression and co-morbid PD, ${ }^{17}$ some IAPT providers refuse to treat this patient group on the grounds that they deem the patient's mental health to be too serious and/ or complex for them to be able to safely and effectively treat them. According to the findings of our study, the net effect of this has been that GPs end up having to either encourage enthusiasm for a referral, by stressing the patient's simpler problems, or, when faced with a rejected referral for a patient needing treatment, writing-up, or embellishing descriptions of patients' risk status in order to ensure the patient is seen by secondary care services. 
Our findings also show that when patients with PD fall in the gap between these two services, GPs are faced with sourcing alternative treatment providers privately or in the third sector.

Findings from this study also suggest that GPs wanted IAPT services to offer more specialised treatments for patients with PD and depression in order to see improved treatment outcomes. Indeed, a recent study of over 1000 IAPT attenders found that the presence of personality difficulties at baseline in patients with depression independently predicted smaller treatment gains and lower likelihood of recovery at the end of the treatment. ${ }^{26}$ These findings were not explained by differential treatment drop-out, differences in severity of depression/ anxiety at the beginning of treatment or by differences in the number of sessions attended. ${ }^{26}$ They, therefore, suggest that if IAPT services are to treat patients with depression and/or anxiety and co-morbid PD, there is a need for them to provide a more personalised treatment to this patient group to achieve the same treatment gains experienced by patients without personality pathology.

\section{Study strengths and weaknesses}

This is the first study to detail GPs' views and experiences of managing patients with PD, and their views on IAPT services in relation to meeting the mental health needs of these patients. Data were collected using in-depth interviews that enabled GPs to raise issues that were salient to them. The GPs interviewed were able to refer patients with PDs and co-morbid depression and/or anxiety to local IAPT services, but not all IAPT services in the UK accept these patients. Thus, our participants may have held views about the role of IAPT services that differ from those held by GPs working in other areas. GPs recruited to the study worked in practices based in the West of England. The majority of GPs interviewed were based in practices serving relatively affluent patient populations. This might limit the generalisability of our findings; although there were no clear differences between the accounts given by GPs based in these practices and those based in practices serving more deprived patient populations, suggesting our findings were robust. Nonetheless, many of the key findings were evident across all the interviews, suggesting that they may be relevant to other GPs, and data saturation was reached. As GPs do not formally diagnose patients with PD, it may have been that during the interviews they talked about patients who they viewed as having this condition, but who did not have a formal diagnosis.

\section{Conclusions and implications}

GPs consider patients with PD to have complex needs manifesting in challenging consultations that require careful management. GPs also report finding it difficult to monitor patients' mental health and risk of selfharm. Our findings also suggest that GPs do not consider IAPT services as able to effectively treat patients with depression and/or anxiety and co-morbid PD, and more specifically designed treatments, delivered by skilled therapists, are needed. Patients with PD have a propensity to fall between primary and secondary care services, and currently, it seems no health service is able to 'hold' and provide long-term risk management for this patient group. As such, GPs are currently having to support the treatments needs of this patient group with limited guidance on how best to treat them.

Acknowledgements We would like to thank all the GPs who agreed be interviewed in this study.

Contributors KMT was the principal investigator for this qualitative study. LF conducted and analysed the interviews on which the paper is based and led on writing this manuscript. PM, NW, DK, KT and LF contributed to the design of the study and editing of the final manuscript and the refining of its intellectual content.

Funding This work was funded by the National Institute for Health Research (NIHR) School for Primary Care Research. This study was supported by the NIHR Biomedical Research Centre at University Hospitals Bristol NHS Foundation Trust and the University of Bristol. The views expressed in this publication are those of the authors and not necessarily those of the NHS, the NIHR or the Department of Health.

Competing interests None declared.

Patient consent for publication Not required.

Ethics approval Ethical approval to conduct the study was given by NRES Committee South West-Exeter (reference: 16/SW/0229).

Provenance and peer review Not commissioned; externally peer reviewed.

Data sharing statement The datasets analysed during the current study are not publicly available, as participants were not asked to consent to this at the time of data collection. However, if requests for data sharing are made, the University of Bristol Data Access Committee will consider them and decide whether or not they can be met.

Open access This is an open access article distributed in accordance with the Creative Commons Attribution Non Commercial (CC BY-NC 4.0) license, which permits others to distribute, remix, adapt, build upon this work non-commercially, and license their derivative works on different terms, provided the original work is properly cited, appropriate credit is given, any changes made indicated, and the use is non-commercial. See: http://creativecommons.org/licenses/by-nc/4.0/.

\section{REFERENCES}

1. American Psychiatric Association. Diagnostic and statistical manual of mental disorders (DSM-5®): American Psychiatric Pub, 2013.

2. Coid J, Yang M, Tyrer P, et al. Prevalence and correlates of personality disorder in Great Britain. Br J Psychiatry 2006;188:423-31.

3. Moran P, Jenkins R, Tylee A, et al. The prevalence of personality disorder among UK primary care attenders. Acta Psychiatr Scand 2000;102:52-7.

4. Newton-Howes G, Tyrer P, Anagnostakis K, et al. The prevalence of personality disorder, its comorbidity with mental state disorders, and its clinical significance in community mental health teams. Soc Psychiatry Psychiatr Epidemiol 2010;45:453-60.

5. Moran P, Romaniuk H, Coffey $\mathrm{C}$, et al. The influence of personality disorder on the future mental health and social adjustment of young adults: a population-based, longitudinal cohort study. Lancet Psychiatry 2016;3:636-45.

6. Gunderson JG, Stout RL, McGlashan TH, et al. Ten-year course of borderline personality disorder: psychopathology and function from the Collaborative Longitudinal Personality Disorders study. Arch Gen Psychiatry 2011;68:827-37.

7. Moran P, Stewart R, Brugha T, et al. Personality disorder and cardiovascular disease: results from a national household survey. $J$ Clin Psychiatry 2007;68:69-74.

8. Fok M, Hotopf M, Stewart R, et al. Personality disorder and self-rated health: a population-based cross-sectional survey. J Pers Disord 2014;28:319-33.

9. Fok ML, Hayes RD, Chang CK, et al. Life expectancy at birth and all-cause mortality among people with personality disorder. $J$ Psychosom Res 2012;73:104-7. 
10. Tyrer P, Mulder R, Crawford M, et al. Personality disorder: a new global perspective. World Psychiatry 2010;9:56-60.

11. Knudsen AK, Skogen JC, Harvey SB, et al. Personality disorders, common mental disorders and receipt of disability benefits: evidence from the British National Survey of Psychiatric Morbidity. Psychol Med 2012;42:2631-40.

12. Newton-Howes G, Weaver T, Tyrer P. Attitudes of staff towards patients with personality disorder in community mental health teams. Aust N Z J Psychiatry 2008;42:572-7.

13. Lewis G, Appleby L. Personality disorder: the patients psychiatrists dislike. Br J Psychiatry 1988;153:44-9.

14. Gask L, Evans M, Kessler D. Personality disorder. BMJ 2013:347:f5276.

15. Tyrer P, Reed GM, Crawford MJ. Classification, assessment, prevalence, and effect of personality disorder. Lancet 2015;385:717-26.

16. French LRM, Turner KM, Dawson S, et al. Psychological treatment of depression and anxiety in patients with co-morbid personality disorder: a scoping study of trial evidence. Personal Ment Health 2017;11:101-17.

17. Department of Health. Talking therapies: a four-year plan of action. 2011.
18. NHS Digital. Improving Access to Psychological Therapies Report, April 2016 Final, May 2016 Primary and Quarter 4 2015/16. 2016.

19. Gyani A, Shafran R, Layard R, et al. Enhancing recovery rates: lessons from year one of IAPT. Behav Res Ther 2013;51:597-606.

20. Sturges JE, Hanrahan KJ. Comparing telephone and face-to-face qualitative interviewing: a research note. Qual Res 2004;4:107-18.

21. Braun V, Clarke V. Using thematic analysis in psychology. Qual Res Psychol 2006;3:77-101.

22. Castleberry A. NVivo 10 [software program]. Version 10: QSR International, 2012.

23. Groves JE. Taking care of the hateful patient. Personality Disorder the Definitive Reader 1978:52-63.

24. Dubovsky AN, Kiefer MM. Borderline personality disorder in the primary care setting. Med Clin North Am 2014;98:1049-64.

25. Bateman AW, Gunderson J, Mulder R. Treatment of personality disorder. Lancet 2015;385:735-43.

26. Goddard E, Wingrove J, Moran P. The impact of comorbid personality difficulties on response to IAPT treatment for depression and anxiety. Behav Res Ther 2015;73:1-7. 